\title{
ORIGINAL ARTICLE/CME ARTICLE
}

\section{Regadenoson pharmacologic stress for myocardial perfusion imaging: A three-way comparison between regadenoson administered at peak exercise, during walk recovery, or no-exercise}

\author{
Randall C. Thompson, MD, , ${ }^{\mathrm{a} b}$ Harshal Patil, MD, ${ }^{\mathrm{b}}$ Elaine C. Thompson, BA, ${ }^{\mathrm{a}}$ \\ Gregory S. Thomas, MD, MPH, ${ }^{\mathrm{c}}$ Mohammed Al-Amoodi, MD, ${ }^{\mathrm{d}}$ \\ Kevin F. Kennedy, MS, ${ }^{b}$ Kevin A. Bybee, MD, ${ }^{a, b}$ A. Iain McGhie, MD, ${ }^{a, b}$ \\ James H. O'Keefe Jr., MD, ${ }^{a, b}$ Lisa Oakes, BSN, ${ }^{a}$ and Timothy M. Bateman, $M D^{a, b}$
}

Background. Regadenoson (Reg) is being administered with increasing frequency either at peak exercise (ExPeak-Reg) or during a slow-down/walking recovery state (ExRec-Reg) rather than at rest (Rest-Reg). The aim of this study was to compare the clinical response of ExPeakReg, ExRec-Reg, and Rest-Reg.

Methods. We compared 531 patients divided equally between Rest-Reg, ExPeak-Reg, and ExRec-Reg matched for age, sex, and BMI.

Results. The average systolic blood pressure (SBP) rise following Reg was modest, but there was considerable heterogeneity and the ExPeak-Reg group had a higher percentage of patients who had a SBP rise of $40 \mathrm{~mm} \mathrm{Hg}$ or a fall of $20 \mathrm{~mm} \mathrm{Hg}$ than either the ExRec-Reg or the Rest-Reg groups $(\geq 40 \mathrm{~mm} \mathrm{Hg}$ rise $6.8 \%, 1.7 \%$, and $1.7 \%$, respectively) $(P<.02)$ $(\geq 20 \mathrm{~mm} \mathrm{Hg}$ fall $15.8 \%, \mathbf{1 3 . 0 \%}$, and $7.3 \%$, respectively) $(P<.05)$. Chest discomfort, nausea, dizziness, and interfering abdominal radiotracer activity were less common in both exercise Reg groups compared to Rest-Reg $(P<.05)$.

Conclusion. Regadenoson injected at peak of symptom-limited exercise was generally well tolerated, but some patients had a significant rise or drop in SBP. There is no apparent advantage of administering regadenoson at peak exercise rather than during walk recovery, and the latter approach may have a greater safety margin. (J Nucl Cardiol 2013;20:214-21.)

Key Words: Exercise $\bullet$ stress testing $\bullet$ pharmacologic stress $\bullet$ A2A adenosine receptor agonists

From the Saint-Luke's Mid America Heart Institute, ${ }^{a}$ Kansas City, MO; University of Missouri-Kansas City School of Medicine, ${ }^{\mathrm{b}}$ Kansas City, MO; Long Beach Memorial Medical Center ${ }^{\mathrm{c}}$ Long Beach, CA; and John Stronger Hospital of Cook County, ${ }^{\mathrm{d}}$ Chicago, IL.

Received for publication Aug 27, 2012; final revision accepted Nov 20, 2012.

Reprint requests: Randall C. Thompson, MD, Saint-Luke's Mid America Heart Institute, 4330 Wornall Road, Suite 2000, Kansas City, MO 64111; rthompson@cc-pc.com, rthomspon@saintlukes.org.

$1071-3581 / \$ 34.00$

Copyright (C) 2012 American Society of Nuclear Cardiology.

doi:10.1007/s12350-012-9660-4

\section{INTRODUCTION}

In clinical practice, pharmacologic stress testing with adenosine or dipyridamole is sometimes combined with either low level or symptom-limited exercise for myocardial perfusion imaging (MPI). ${ }^{1}$ This approach is safe and, compared with vasodilator testing at rest, offers potential advantages of lesser pharmacologic stress-agent side effects and improved image quality. ${ }^{2-7}$ Also, the addition of symptom-limited exercise offers incremental prognostic data which is complementary to the MPI results. ${ }^{4}$ The selective A2a receptor agonist regadenoson was introduced in 2008 and is now the most widely used agent for pharmacologic stress MPI in the United States..$^{8-10}$ It is administered as a bolus and can be conveniently injected in combination with 
exercise stress testing, either in conjunction with planned pharmacologic stress or after a patient undergoes exercise testing and fails to achieve an adequate heart rate response. While there are two reports of the combination of regadenoson with low-level exercise and this experience appears to be favorable, the clinical response of regadenoson combined with full, symptomlimited exercise is not well described. ${ }^{5,11}$ Post-marketing surveillance data identified that some patients have an excessive hypertensive response to regadenoson and a warning about this was subsequently placed in the manufacturer's package insert, particularly for patients with underlying hypertension or undergoing combined low-level exercise. Regadenoson administration combined with full, symptom-limited exercise is being performed with increasing frequency and one concern has been that this combination might result in an excessive hypertensive blood pressure response.

The aim of this study was to determine the clinical response, especially systolic blood pressure (SBP), with regadenoson administered either at the peak of exercise (ExPeak-Reg) or during walk recovery (ExRec-Reg) compared to regadenoson administered at rest (RestReg), in patients clinically referred for stress MPI for the assessment of known or suspected CAD.

\section{METHODS}

\section{Study Design}

This is a case-matched retrospective analysis of 531 elective out-patients and hospital in-patients who had clinically indicated MPI between January 2009 and December 2011. Using Saint Luke's Health System (Kansas City, Missouri) electronic medical record, patient's charts were reviewed after obtaining approval from the Institutional Review Board. The study population was divided into three groups. Group 1 consisted of 177 matched control patients who underwent Reg stress without exercise (Rest-Reg). Group 2 consisted of 177 patients who had Reg injected at peak exercise (ExPeak-Reg). Group 3 consisted of 177 patients who had Reg injected during walk recovery at a reduced treadmill speed $(1.7 \mathrm{mph})$ and no incline, about 2 minutes after symptom-limited exercise (ExRec-Reg). During the phase in of regadenoson in our laboratory, experience was gained using several different protocols. This permitted us the opportunity to retrospectively assess various aspects of efficacy and safety using casematching methodology Patients were referred for pharmacological testing and underwent the combined exercise vasodilator stress test per clinical protocol, or were tested initially with exercise and were given Reg either near-peak exercise or during walk recovery after being unable to achieve adequate heart rate response. Control patients had undergone supine Reg MPI during a similar time period. Symptoms were recorded by the registered nurse supervising the examination.
Patients in the three groups were matched for age, sex, and body mass index (BMI). HR, blood pressures, incidences of rhythm abnormalities, usage of aminophylline, and duration of monitoring were compared between the groups. We also compared rates of reversible myocardial perfusion defects assessed by the 17 -segment model. ${ }^{12}$ In order to determine if the addition of exercise impacts image quality in reference to abdominal radiotracer activity, 146 patients who had been randomly selected (by KFK) were reviewed and assessed in a blinded read by consensus of two readers (RCT and HP).

\section{Data Collection}

Patients were identified from the clinical database at our institution of those undergoing pharmacologic stress testing between January 1, 2009 and December 31, 2011. Individuals in each of the three groups were selected if there was an appropriate age-sex-BMI-matched patient in each of the other two groups. Stress test records and electronic medical records were reviewed to determine medications taken prior to the stress test, adverse reactions (flushing, dyspnea, headache, and nausea), other symptoms, and hemodynamic responses during testing. Blood pressure and HR at rest, before, and after administration of Reg were collected. Treadmill speed and grade, peak and lowest blood pressure, peak HR, peak HR minus resting $\mathrm{HR}$, and percentage of predicted maximum heart rate achieved were also recorded. The presence of AV block, atrial fibrillation, atrial flutter, and ventricular arrhythmias during and after the stress test were recorded by the supervising cardiologists.

\section{Rest and Stress Imaging Protocols}

All images were acquired according to the American Society of Nuclear Cardiology (ASNC) guidelines using Gated SPECT stress only, single-day, or 2-day technetium-99m tracer (sestamibi) protocols. ${ }^{13}$ The patients in both ExPeak-Reg and ExRec-Reg group failed to achieve $\geq 85 \%$ of maximum predicted HR and/or $\geq 5$ METS on a treadmill. Patients were instructed to discontinue caffeine-containing products, calcium channel blockers and nitrates for $24 \mathrm{~h}$ before the test unless clinically contraindicated. Beta blockers were held or administered according to referring physician discretion.

Rest SPECT MPI was performed 45-90 minutes after the administration of technetium-99m sestamibi. For all stress SPECT MPI, 400-mcg of Reg was given as an IV bolus dose into a peripheral vein over approximately 10 seconds immediately followed by a $5-\mathrm{mL}$ saline flush in accordance with ASNC guidelines and the manufacturer's package insert. ${ }^{1}$ 99mTc-Sestamibi was administered approximately 30 seconds after the initiation of the injection of Reg and was followed immediately by a $5-\mathrm{mL}$ saline flush. Gated SPECT MPI was acquired 45-90 minutes after radiotracer administration. The weight/BMI-related $99 \mathrm{mTc}$-sestamibi dose ranged from 8.5 to $11.6 \mathrm{mCi}$ for rest SPECT to 29.5 to $42 \mathrm{mCi}$ for stress SPECT. For 2-day protocols, the stress dose was used for both the rest and the stress portions of the study. 
For patients in Rest-Reg group, Reg was administered without prior exercise. In ExPeak-Reg group, patients performed symptom-limited treadmill exercise and received Reg immediately at peak exercise. These patients then continued exercising for at least 1 minute prior to the initiation of the recovery phase. In ExRec-Reg group, following an inadequate exercise test, patients walked for an additional 2-3 minutes on the treadmill. The treadmill was slowed to approximately $1.7 \mathrm{mph}$ and the incline reduced toward $0 \%$. Reg was administered at approximately 2 minutes of walk recovery; and subjects continued to walk for an additional 2 minutes after the administration of Reg.

ECGs were obtained at baseline and every 2 minutes following Reg infusion for a total of 4 minutes post-Rest-Reg and 6 minutes post-exercise, or longer in cases with persistent symptoms or instability. Horizontal or down-sloping ST segment depression of $1 \mathrm{~mm}$ or up-sloping of $1.5 \mathrm{~mm}$ at $80 \mathrm{~ms}$ after the $\mathrm{J}$ point was considered positive. A 17 -segment model was used to count the number of segments with reversible defects. ${ }^{12}$ Segments were considered to have a reversible defect if the stress perfusion score was greater than the rest score.

\section{Statistical Analysis}

For comparison of the three groups, continuous variables were compared using one-way analysis of variance and categorical variable compared using Fisher's exact test. Multivariable logistic regression analysis was used to find predictors of exaggerated low and high blood pressure responses. All the groups were matched on three important characteristics (age, gender, and BMI). However, to account for additional confounding variables, we performed multivariable adjusted general linear model to predict the outcomes of interest (HR, blood pressure). The potential adjustments were beta-blocker (BB) and calcium channel blocker (CCB) use, age, presence of ischemia, and previous hypertension. If outcome variables were dichotomized in a high/low, we then followed current recommendations on $10 \mathrm{EPV}$ (1 adjustment variable per 10 events) to avoid overfitting of our model. ${ }^{13}$ The three groups were our main predictor variable, and we compared all 2-way comparisons using a Bonferroni adjustment for multiple comparisons. A $P$ value of .05 was considered statistically significant, and all analyses were performed with SAS version 9.2 (Cary, NC).

\section{RESULTS}

\section{Baseline Characteristics}

177 patients each in ExPeak-Reg and ExRec-Reg groups were matched with 177 Rest-Reg controls for age, sex, and BMI. The average age of the study population was $59.7 \pm 9.8$ years, $72.5 \%$ were male and average BMI of $30.1 \pm 5.5 \mathrm{~kg} / \mathrm{m}^{2}$ The frequency of diabetes mellitus (DM), hypertension, family history of coronary artery disease (CAD), and smokers were similar, while history of prior CAD and hyperlipidemia was more common in ExPeak-Reg and ExRec-Reg groups than control (Table 1). The number of patients taking calcium channel blockers was not different while beta blocker use was more common in Exercise-Reg groups, especially the ExPeak-Reg group. The number of patients with abnormal perfusion as expressed by SSS, SRS, and SDS was not statistically different between the three groups.

\section{Exercise and Hemodynamic Responses}

The mean level of exercise achieved in the ExPeakReg group was higher than in the ExRec-Reg group $(7.8 \pm 2.6$ vs $7.2 \pm 2.2 \mathrm{MET}$, respectively) $(P<.05)$. By protocol, the treadmill speed and incline at the time of injection of regadenoson were higher for the ExPeakReg group compared to the ExRec-Reg groups (speed$2.7 \pm 0.8$ vs $1.9 \pm 0.5 \mathrm{mph}$, and incline $-10.6 \% \pm 4.9 \%$ vs $1.4 \% \pm 3.0 \%$ grade, respectively). The baseline HR and SBP and diastolic blood pressure (DBP) were slightly lower in the exercise groups than in the RestReg group (Table 2).

As expected, heart rate increased with exercise. Following Reg administration, the additional rise in HR was less for both the exercise groups than for the RestReg group (5.4 \pm 10.6 bpm ExPeak-Reg, $0.9 \pm 9.6$ ExRec-Reg vs $8.6 \pm 9.1 \quad$ BPM Rest-Reg group) $(P<.001)$. Also, there were more patients with a rise in heart rate of $>15 \%$ in the Rest-Reg group (32.2\%) than the two exercise groups $(13 \%$ and $6.2 \%)(P<.001)$.

Immediately after Reg administration, on average SBP increased slightly in both of the exercise groups (4.6 and $0.1 \mathrm{~mm} \mathrm{Hg}$, respectively) and fell slightly in the RestReg group $(-1.6 \mathrm{~mm} \mathrm{Hg})$. Average DBP fell slightly immediately following Reg administration in all three groups. However, there was considerable heterogeneity of blood pressure response. In particular, the ExPeak-Reg group had a higher percentage of patients who had a SBP rise of 40 or $20 \mathrm{~mm} \mathrm{Hg}$, or a fall of $20 \mathrm{~mm} \mathrm{Hg}$ after regadenoson, than either the Rest-Reg group or the ExRec-Reg group $(\geq 40 \mathrm{~mm} \mathrm{Hg}$ rise $6.8 \%, 1.7 \%$, and $1.7 \%$, respectively) $(P<.02)(\geq 20 \mathrm{~mm} \mathrm{Hg}$ rise $21.5 \%$, $6.2 \%$, and $9.6 \%$, respectively) $(P<.001)(\geq 20 \mathrm{~mm} \mathrm{Hg}$ fall $15.8 \%, 13.0 \%$, and $7.3 \%$, respectively) $(P<.05)$. The patients in ExRec-Reg group were less likely to have a large rise or fall in SBP after Reg administration than the patients in the ExPeak-Reg group (for two-way comparison: $P<.05$ for $\geq 40 \mathrm{~mm} \mathrm{Hg}$ blood pressure rise, $P<$ .003 for $\geq 20 \mathrm{~mm} \mathrm{Hg}$ blood pressure rise, and $P<.02$ for $\geq 20 \mathrm{~mm} \mathrm{Hg}$ blood pressure fall) (Table 2).

Compared to patients who did not have a significant blood pressure rise, patients who had a $>20 \mathrm{~mm} \mathrm{Hg}$ rise in 
Table 1. Characteristics and stress test results of patients in the three groups

\begin{tabular}{llllr}
\hline & $\begin{array}{c}\text { Rest-Reg } \\
\mathbf{n}=\mathbf{1 7 7}\end{array}$ & $\begin{array}{c}\text { ExPeak-Reg } \\
\mathbf{n}=\mathbf{1 7 7}\end{array}$ & $\begin{array}{c}\text { ExRec-Reg } \\
\mathbf{n}=\mathbf{1 7 7}\end{array}$ & $\boldsymbol{P}$ value \\
\hline Age & $59.7 \pm 9.9$ & $59.7 \pm 9.8$ & $60.2 \pm 9.7$ & .92 \\
Male & $129(72.9 \%)$ & $129(72.9 \%)$ & $129(72.9 \%)$ & 1.00 \\
BMI & $30.1 \pm 5.4$ & $30.2 \pm 5.5$ & $29.9 \pm 4.8$ & .94 \\
Diabetes & $47(26 \%)$ & $44(24 \%)$ & $49(27 \%)$ & .86 \\
Hypertension & $130(73.4 \%)$ & $134(75.7 \%)$ & $132(74.6 \%)$ & .49 \\
Family Hx of CAD & $86(48.6 \%)$ & $92(52.0 \%)$ & $79(44.6 \%)$ & .33 \\
Smoker & $55(31.1 \%)$ & $43(24.3 \%)$ & $38(21.5 \%)$ & .11 \\
Calcium channel blockers & $13(7.3 \%)$ & $8(4.5 \%)$ & $16(9.0 \%)$ & .37 \\
Beta-blockers & $32(18.1 \%)$ & $54(30.5 \%)$ & $35(19.8 \%)$ & .012 \\
Known CAD & $64(36.2 \%)$ & $80(45.2 \%)$ & $89(50.3 \%)$ & .025 \\
Hyperlipidemia & $127(71.8 \%)$ & $146(82.5 \%)$ & $142(80.2 \%)$ & .039 \\
SSS & $3.5 \pm 9.4$ & $2.6 \pm 4.5$ & $2.8 \pm 5.2$ & .38 \\
SRS & $1.3 \pm 3.0$ & $1.4 \pm 3.3$ & $2.3 \pm 6.7$ & .11 \\
SDS & $2.2 \pm 8.1$ & $1.4 \pm 2.9$ & $1.0 \pm 6.5$ & .24 \\
Ischemic nuclear response & $31(17.5 \%)$ & $39(22.0 \%)$ & $38(21.5 \%)$ & .516 \\
Non-diagnostic or equivocal scan & $13(7.3 \%)$ & $5(2.8 \%)$ & $4(2.3 \%)$ & .031 \\
ECG response & & & & $<.001$ \\
Ischemic & $1(0.6 \%)$ & $18(10.2 \%)$ & $26(14.7 \%)$ & $13(7.3 \%)$ \\
Non-diagnostic & $24(13.6 \%)$ & $31(17.6 \%)$ & $138(78.0 \%)$ & \\
Non-ischemic & $151(85.8 \%)$ & $127(72.2 \%)$ & & \\
\hline
\end{tabular}

blood pressure after regadenoson had a slightly higher BMI, were more likely to have experienced nausea, and received Reg at a higher treadmill speed and incline (Table 3).

\section{Symptoms and Adverse Reactions}

Combining Reg with symptom-limited exercise was well tolerated and there were no major adverse events. Symptoms of chest discomfort, nausea, and dizziness were less common in the exercise groups than matched patients undergoing Reg stress without exercise (Table 3). Dizziness did not seem to be related to blood pressure changes as it was not recorded more commonly in the patients who had increases or decreases of SBP $>20 \mathrm{~mm} \mathrm{Hg}$. Shortness of breath was commonly reported in all groups, but was somewhat more common in the ExRec-Reg group. No patients developed heart block in any group. The frequency of development of premature atrial and ventricular complexes, ventricular triplets, or administration of aminophylline was not significantly different among the three groups. Prolonged monitoring post-exercise completion was considered to be a possible sensitive marker for exaggerated symptoms, dynamic ECG changes, or hemodynamic instability. Prolonged monitoring beyond routine protocol (defined as $>4$ minutes in the Rest-Reg group or $>7$ minutes in the exercise groups) was more frequent in the ExPeak-Reg group (11.9\%) compared with the RestReg group $(2.8 \%)(P<.004)$ or the ExRec-Reg group $(8.5 \%)(P=\mathrm{ns})$. Analysis of several patient characteristics found that BMI was the only identifiable predictor of a rise in blood pressure of $>20 \mathrm{~mm} \mathrm{hg}$ (Table 4).

\section{Image Quality}

The number of patients with abdominal radiotracer activity overlapping the heart was less in both the exercise groups than in the Rest-Reg group. Seventeen patients (35.4\%) in the Rest-Reg group had abdominal radiotracer activity overlapping at least one left ventricular segment compared to 8 patients (16\%) of the ExPeak-Reg group and 7 (14.6\%) of the ExRec-Reg group $(P=.029)$. The number of patients with at least some adverse impact of abdominal radiotracer activity on diagnostic image quality was also less in the exercise groups than in the Rest-Reg group. Twentyfive patients (32.1\%) of the Rest-Reg group had adverse impact on diagnostic quality compared to $11(22 \%)$ of the ExPeak-Reg group and 10 (20.8\%) of the ExRec-Reg group $(P=.001)$. The number of patients in each group who had a 
Table 2. Heart rate, blood pressure, and exercise response of patients in the three groups

\begin{tabular}{|c|c|c|c|c|}
\hline & $\begin{array}{l}\text { Rest-Reg } \\
n=177\end{array}$ & $\begin{array}{c}\text { ExPeak-Reg } \\
n=177\end{array}$ & $\begin{array}{c}\text { ExRec-Reg } \\
n=177\end{array}$ & $P$ value \\
\hline HR immediately before Reg injection & $89.5 \pm 19.4$ & $113.1 \pm 15.7$ & $122.5 \pm 18.3$ & $<.001$ \\
\hline HR after Reg injection & $98.1 \pm 16.5$ & $118.5 \pm 17.1$ & $123.4 \pm 18.7$ & $<.001$ \\
\hline Delta HR after Reg injection & $8.6 \pm 9.1$ & $5.4 \pm 10.6$ & $0.9 \pm 9.6$ & $<.001$ \\
\hline Peak HR & $92.2 \pm 17.2$ & $123.1 \pm 16.4$ & $128.3 \pm 17.3$ & $<.001$ \\
\hline HR increase $15 \%$ after Reg & $57(32.2 \%)$ & $23(13.0 \%)$ & $11(6.2 \%)$ & $<.001$ \\
\hline SBP at rest & $138.7 \pm 20.7$ & $131.2 \pm 18.1$ & $129.9 \pm 17.4$ & $<.001$ \\
\hline SBP before Reg injection & $132.6 \pm 20.4$ & $149.8 \pm 22.7$ & $154.6 \pm 22.3$ & $<.001$ \\
\hline SBP after Reg injection & $131.0 \pm 22.1$ & $154.4 \pm 28.6$ & $154.5 \pm 26.0$ & $<.001$ \\
\hline $\mathrm{SBP}>200$ at time of injection & $0(0.0)$ & $6(3.4 \%)$ & 7 (4.0\%) & .017 \\
\hline SBP Rise by $40 \mathrm{~mm}$ Hg post-Reg & $3(1.7 \%)$ & $12(6.8 \%)$ & $3(1.7 \%)$ & .020 \\
\hline SBP Rise by $20 \mathrm{~mm}$ Hg post-Reg & $11(6.2 \%)$ & $38(21.5 \%)$ & $17(9.6 \%)$ & $<.001$ \\
\hline SBP Fall by 20 mm Hg post-Reg & $23(13.0 \%)$ & $28(15.8 \%)$ & $13(7.3 \%)$ & .039 \\
\hline Peak SBP & $131.5 \pm 22.4$ & $156.1 \pm 28.6$ & $156.5 \pm 26.5$ & $<.001$ \\
\hline DBP at rest & $81.3 \pm 11.3$ & $76.7 \pm 9.5$ & $76.6 \pm 9.3$ & $<.001$ \\
\hline DBP before Reg injection & $76.9 \pm 12.9$ & $77.6 \pm 14.1$ & $78.9 \pm 11.3$ & .338 \\
\hline DBP after Reg injection & $74.8 \pm 12.4$ & $75.5 \pm 12.1$ & $77.7 \pm 12.5$ & .078 \\
\hline Peak DBP & $75.6 \pm 12.7$ & $75.6 \pm 12.4$ & $77.6 \pm 10.7$ & .182 \\
\hline Delta DBP & $-9.5 \pm 51.3$ & $-1.3 \pm 12.4$ & $0.8 \pm 10.5$ & .005 \\
\hline METS achieved & $0.0 \pm 0.1$ & $7.8 \pm 2.6$ & $7.2 \pm 2.2$ & $<.001$ \\
\hline Speed before Reg injection (MPH) & $0.0 \pm 0.0$ & $2.6 \pm 0.8$ & $1.9 \pm 0.5$ & $<.001$ \\
\hline Incline before Reg injection (\% grade) & $0.0 \pm 0.0$ & $10.5 \pm 5.0$ & $1.4 \pm 3.0$ & $<.001$ \\
\hline Duration of monitoring & $4.1 \pm 0.9$ & $6.6 \pm 2.2$ & $6.6 \pm 1.9$ & - \\
\hline Percentage of Max HR & $57.7 \pm 10.9$ & $76.9 \pm 9.8$ & $80.6 \pm 12.0$ & $<.001$ \\
\hline
\end{tabular}

Table 3. Side-effect profiles among the three groups

\begin{tabular}{lllll}
\hline & $\begin{array}{c}\text { Rest-Reg } \\
\mathbf{n}=\mathbf{1 7 7}\end{array}$ & $\begin{array}{c}\text { ExPeak-Reg } \\
\mathbf{n = 1 7 7}\end{array}$ & $\begin{array}{c}\text { ExRec-Reg } \\
\mathbf{n}=\mathbf{1 7 7}\end{array}$ & P value \\
\hline Chest pain & $48(27.1 \%)$ & $26(14.7 \%)$ & $38(21.5 \%)$ & .015 \\
Nausea & $33(18.6 \%)$ & $8(4.5 \%)$ & $20(11.3 \%)$ & $<.001$ \\
Vomiting & $1(0.6 \%)$ & $0(0.0 \%)$ & $1(0.6 \%)$ & ns \\
Flushing & $16(9.0 \%)$ & $5(2.8 \%)$ & $16(9.0 \%)$ & .020 \\
Dizziness & $57(32.2 \%)$ & $27(15.3 \%)$ & $20(11.3 \%)$ & $<.001$ \\
SOB & $102(57.6 \%)$ & $105(59.7 \%)$ & $154(87.0 \%)$ & $<.001$ \\
PACs & $6(3.4 \%)$ & $6(3.4 \%)$ & $5(2.8 \%)$ & 1.000 \\
PVCs & $9(5.1 \%)$ & $12(6.8 \%)$ & $19(10.8 \%)$ & .116 \\
Ventricular triplet & $0(0.0 \%)$ & $0(0.0 \%)$ & $0(0.0 \%)$ & NA \\
2nd or 3rd degree AV block & $0(0.0 \%)$ & $0(0.0 \%)$ & $0(0.0 \%)$ & NA \\
Prolonged monitoring (Rest-Reg $>4$ min, & $5(2.8 \%)$ & $21(11.9 \%)$ & $15(8.5 \%)$ & .004 \\
$\quad$ exercise $>7$ min) & $4.1 \pm 0.9$ & $6.6 \pm 2.2$ & $6.6 \pm 1.9$ & NA \\
Duration of monitoring & & & &
\end{tabular}

non-diagnostic or equivocal scintigraphic response by clinical read is shown in Table 1 . Significantly more patients in the Rest-Reg group had a non-diagnostic or equivocal scan than in either of the exercise groups.

\section{DISCUSSION}

This study describes the results of two protocols combining regadenoson with symptom-limited exercise, 
Table 4. Analysis of potential predictors of blood pressure rise of $>20 \mathrm{~mm} \mathrm{Hg}$ after regadenoson administration

SBP Rise by $20 \mathrm{~mm} \mathrm{Hg}$ post-regadenoson

\begin{tabular}{llll} 
& \multicolumn{1}{c}{$\begin{array}{c}\text { Yes } \\
\mathbf{n}=\mathbf{6 6}\end{array}$} & \multicolumn{1}{c}{$\mathbf{N o}$} & \\
\hline Rest-Reg & $11(6 \%)$ & $\mathbf{n} \mathbf{4 6 5}$ & $\boldsymbol{P}$ Value \\
ExPeak-Reg & $38(21 \%)$ & $166(94 \%)$ & $<.001$ \\
ExRec-Reg & $17(10 \%)$ & $160(79 \%)$ & \\
Age & $59.7 \pm 9.5$ & $59.9 \pm 9.9$ & .914 \\
Male & $44(66.7 \%)$ & $343(73.8 \%)$ & .238 \\
BMI & $31.7 \pm 6.6$ & $29.8 \pm 5.0$ & .006 \\
Hypertension & $54(81.8 \%)$ & $342(73.5 \%)$ & .175 \\
DM & 66 & 465 & .800 \\
Known CAD & $27(40.9 \%)$ & $206(44.3 \%)$ & .691 \\
Smoker & $12(18.2 \%)$ & $124(26.7 \%)$ & .175 \\
Baseline SBP & $133.1 \pm 19.8$ & $133.3 \pm 19.3$ & .935 \\
Baseline DBP & $80.6 \pm 12.4$ & $79.5 \pm 31.6$ & .769 \\
Use of BB & $18(27.3 \%)$ & $103(22.2 \%)$ & .350 \\
Use of CCA & $6(9.1 \%)$ & $31(6.7 \%)$ & .441 \\
SBP before Reg & $140.4 \pm 17.5$ & $146.4 \pm 24.4$ & .053 \\
\hline
\end{tabular}

against the standard rest protocol, for radionuclide MPI. In this retrospective, case-matched study including a large proportion of patients with pre-existing CAD, administration of a regadenoson bolus of $400 \mathrm{mcg}$ at the peak or during early walk recovery of symptom-limited treadmill testing was well tolerated and improved image appearances. No major adverse events occurred in the 531 subjects studied, including the 354 who underwent combined exercise-regadenoson stress. In a minority of patients tested, there was an exaggerated rise or fall in SBP after the administration of regadenoson and this blood pressure change occurred most frequently in those patients in whom regadenoson was given at peak exercise. This group also more frequently required prolonged monitoring during the recovery phase, suggesting that supervising clinicians more frequently felt the need for extra observation due to exaggerated signs, symptoms, or ECG response.

Previous studies combining low-level exercise (generally 1-2 mph on a motorized treadmill) and vasodilator stress with adenosine, dipyridamole, or regadenoson for MPI have demonstrated that there are fewer vasodilator drug side effects and improved image quality with the combined approach. ${ }^{2-7,11,12}$ Exercise leads to splanchnic artery vasoconstriction and catecholamine release, effects thought to be important in improvement in MPI image quality and symptom mitigation, respectively. ${ }^{14}$ Consistent with these studies, our results showed that symptoms of chest pain, nausea, and dizziness were less frequent in the groups of patients undergoing exercise and that image-interfering abdominal radiotracer activity was less than in the group of patients administered regadenoson at rest. By clinical interpretation there were also fewer non-diagnostic/ equivocal scintigraphic scans in the exercise groups than in the group administered regadenoson at rest.

Symptom-limited exercise combined with dipyridamole for MPI testing was shown by Ahlberg et al to provide incremental prognostic data compared to dipyridamole infused at rest. Part of the rationale for combining regadenoson with symptom-limited exercise is the additional prognostic value of the exercise testing. Although our study did not investigate this aspect, it is reasonable to expect that such prognostic value will be present, as it is with exercise combined with dipyridamole.

Additional rationale for combining regadenoson with symptom-limited exercise has to do with the ease of administering this drug compared to other vasodilator stress agents. Regadenoson is administered as a bolus rather than as a weight-based, timed infusion, and it is quite convenient to give it at the end of a trial of treadmill exercise. For example, if a patient is referred for exercise testing but does not achieve an adequate heart rate response, a dose of regadenoson can be taken from the storage shelf and administered in a matter of seconds, converting the test to pharmacologic stress. Likewise, if a patient is referred for pharmacologic stress testing, exercise 
can be attempted first, or simply performed in combination for its prognostic value. When exercise is attempted first and the patient is able to achieve $85 \%$ of predicted heart rate and an adequate MET level, a pharmacologic stress agent is not needed, thus saving cost. Adenosine and dipyridamole, by contrast, cannot be given as conveniently in this setting because they are administered as 4- or 6minute infusions. Dipyridamole also has a slower onset of action and much longer duration of effect.

Recently Partington et al $^{15}$ described their experience with the use of regadenoson for 211 patients having a suboptimal heart rate response on treadmill exercise testing. Our study confirms the primary finding of the Partington study - that the combination of regadenoson and symptom-limited exercise is feasible and well tolerated and there are fewer symptoms compared with regadenoson stress without exercise. Our study also expands the findings to a larger and more diverse patient population. The patients in the Partington study who received regadenoson with exercise accounted for $30 \%$ of those in their laboratory who had failed to achieve an adequate heart rate response to exercise, and patients with a body weight $<160$ pounds were not included. By contrast, exercise combined with regadenoson is performed more broadly in our laboratory. Our study additionally demonstrated that image quality is improved and the number of non-diagnostic or equivocal scans is reduced when symptom-limited exercise is added to regadenoson stress. However, only $12 \%$ of these patients in the Partington study had a rise in SBP $>10 \mathrm{~mm} \mathrm{Hg}$ after the administration of regadenoson. By contrast, in the group of patients in our study who received regadenoson right at peak exercise, $21.5 \%$ had a rise of $\geq 20 \mathrm{~mm} \mathrm{Hg}$. Regadenoson was administered either at peak exercise or during walking at $1.7 \mathrm{mph}$ in the Partington study. Also, the average achieved MET level was $7.3 \pm 2.3$ their patients compared to $7.8 \pm 2.6$ in our ExPeak-Reg group and the peak heart rate achieved appears to have been lower in their study. Thus, we may have detected more patients with a significant blood pressure rise in this group in our study because regadenoson was given at a higher level of exercise stress.

\section{LIMITATIONS}

This is a single-center, retrospective study and our patients included both those who were initially referred for exercise testing and for pharmacologic stress for MPI. While exercise hemodynamics was recorded per clinical routine, these parameters would be entered more rigorously according to time intervals in a prospective randomized trial. Thus, it is possible that we underestimated blood pressure lability in our groups or that the blood pressure differences among the groups were in part artifactual. Also, we did not include a detailed patient symptom questionnaire and thus have only limited data on the intensity of patient symptoms. For example, dizziness was more frequently recorded as a symptom in the patients administered regadenoson at rest, but the intensity or quality of this symptom was not determined. Dizziness was not recorded more commonly in the patients who had labile blood pressure swings. We also do not have intermediate term followup, although prior studies would suggest that the addition of exercise improves the prognostic discrimination of stress MPI testing.

\section{SUMMARY}

This study demonstrates that exercise combined with regadenoson stress for MPI is well tolerated. Chest pain, dizziness, nausea, and image-interfering abdominal radiotracer activity are less frequent compared to regadenoson administered at rest. Some patients have an exaggerated blood pressure response when regadenoson is administered at the peak of exercise an effect which is less frequent when regadenoson is administered during walk-recovery. There does not appear to be any advantage in administering regadenoson during peak exercise compared with rest-recovery, and there may be a greater safety margin with the latter approach.

\section{References}

1. Henzlova MJ, Cerqueira MD, Hansen CL, Taillefer R, Yao SS. ASNC imaging guidelines for nuclear cardiology procedures; Stress protocols and tracers. J Nucl Cardiol 2009;16. doi: 10.1007/s12350-009-9062-4.

2. Pennell DJ, Mavrogeni SI, Forbat SM, Karwatowski SP, Underwood SR. Adenosine combined with dynamic exercise for myocardial perfusion imaging. J Am Coll Cardiol. 1995;25: 1300-9.

3. Thomas GS, Prill NV, Majmundar H, Fabrizi RR, Thomas JJ, Hayashida $\mathrm{C}$, et al. Treadmill exercise during adenosine infusion is safe, results in fewer adverse reactions, and improves myocardial perfusion image quality. J Nucl Cardiol 2000;7:439-46.

4. Ahlberg AW, Baghdasarian SB, Athar H, Thompsen JP, Katten DM, Noble GL, et al. Symptom-limited exercise combined with dipyridamole stress: Prognostic value in assessment of known or suspected coronary artery disease by use of gated SPECT imaging. J Nucl Cardiol 2008;15:42-56.

5. Thomas GS, Thompson RC, Miyamoto MI, Ip TK, Rice DL, Milikien D, et al. The RegEx trial: A randomized, double-blind, placebo- and active-controlled pilot study combining regadenoson, a selective $\mathrm{A}(2 \mathrm{~A})$ adenosine agonist, with low-level exercise, in patients undergoing myocardial perfusion imaging. J Nucl Cardiol 2009; $16: 63-72$

6. Holly TA, Satran A, Bromet DS, Mieres JH, Frey MJ, Elliott MD, et al. The impact of adjunctive adenosine infusion during exercise myocardial perfusion imaging: Results of the Both Exercise and Adenosine Stress Test (BEAST) trial. J Nucl Cardiol 2003;10:291-6. 
7. Vitola JV, Brambatti JC, Caligaris F, Lesse CR, Nogueira PR, Joaquim AI, et al. Exercise supplementation to dipyridamole prevents hypotension, improves electrocardiogram sensitivity, and increases heart-to-liver activity ratio on Tc-99m sestamibi imaging. J Nucl Cardiol 2001;8:652-9.

8. Hendel RC, Bateman TM, Cerqueira MD, Iskandrian AE, Leppo JA, Blackburn B, et al. Initial clinical experience with regadenoson, a novel selective A2A agonist for pharmacologic stress single-photon emission computed tomography myocardial perfusion imaging. J Am Coll Cardiol 2005;46:2069-75.

9. Iskandrian $\mathrm{AE}$, Bateman $\mathrm{TM}$, Belardinelli L, Blackburn B, Cerqueira MD, Hendel RC, et al. Adenosine versus regadenoson comparative evaluation in myocardial perfusion imaging: Results of the ADVANCE phase 3 multicenter international trial. J Nucl Cardiol 2007; 14:645-58.

10. Cerqueira MD, Nguyen $P$, Staehr P, Underwood SR, Iskandrian AE. Effects of age, gender, obesity, and diabetes on the efficacy and safety of the selective A2A agonist regadenoson versus adenosine in myocardial perfusion imaging integrated ADVANCE-MPI trial results. JACC Cardiovasc Imaging 2008;1:307-16.

11. Kwon DH, Cerqueira MD, Young R, Houghtaling P, Lieber E, Menon V, et al. Lessons from regadenoson and low-level treadmill/regadenoson myocardial perfusion imaging: Initial clinical experience in 1263 patients. J Nucl Cardiol 2010;17:853-7.
12. Cerqueira MD, Weissman NJ, Dilsizian V, Jacobs AK, Kaul S, Laskey WK, et al. Standardized myocardial segmentation and nomenclature for tomographic imaging of the heart: A statement for healthcare professionals from the Cardiac Imaging Committee of the Council on Clinical Cardiology of the American Heart Association. Circulation 2002;105:539-42.

13. Peduzzi P, Concato J, Kemper E, Holford TR, Feinstein AR. A simulation study of the number of events per variable in logistic regression analysis. J Clin Epidemiol 1996;49:1373-9.

14. Rehm PK, Atkins FB, Ziessman HA, Green SE, Akin EA, Fox LM, et al. Frequency of extra-cardiac activity and its effect on 99Tcm-MIBI cardiac SPET interpretation. Nucl Med Commun 1996;17:851-6

15. Partington SL, Lanka V, Hainer J, Blankstein R, Skali H, Foreman $\mathrm{DE}$, et al. Safety and feasibility of regadenoson use for suboptimal heart rate response during symptom-limited standard Bruce exercise stress test. J Nucl Card 2012; doi:10.1007/s12350-012-9562-5 (online first). 